\title{
Transition Metal-Nonmetal in Conductivity of Ceramic Hole-Doped Cobaltites
}

\author{
Yurii Nikolaevich Chiang ${ }^{1}$, Mihail Olegovich Dzyuba ${ }^{1}$, Olga Georgievna Shevchenko ${ }^{1}$, \\ Anatolii Antonovich Kozlovskii ${ }^{2}$, Vitalii Philipovich Khirnyi ${ }^{2}$ \\ ${ }^{1}$ B.Verkin Institute for Low Temperature Physics and Engineering, \\ National Academy of Sciences of Ukraine, Kharkov, Ukraine \\ ${ }^{2}$ Institute of Single Crystals, National Academy of Sciences of Ukraine, \\ Kharkov, Ukraine \\ E-mail: chiang@ilt.kharkov.ua
}

Received September 17, 2010; revised November 8, 2010; accepted October 21, 2010

\begin{abstract}
In bulk granulated cobaltite $\mathrm{La}_{1-\mathrm{x}} \mathrm{Sr}_{\mathrm{x}} \mathrm{CoO}_{3}$ with the size of granules of order of 1 micron at strontium hole doping with replacement factor $x=0.35$, a transition "metal-nonmetal" in the conductivity was revealed, presumably connected with AFM ordering of the moments of granules. The assumption is proved by the agreement between the experiment and results of calculation within the limits of a model offered for electron transport based on the account of in-granule double exchange Zener mechanism and intergranule mechanism of spin-polarized tunneling on the nearest neighbours with AFM exchange interaction. The calculation differs in that conductivities within granules are summarized, while total resistance of the system is represented as a sum of resistances of the granules. In addition, the existence of AFM interaction between granules is supported by the observed insensitivity of conductivity to a low external magnetic field (up to $5 \mathrm{kOe}$ ).
\end{abstract}

Keywords: Granulated Cobaltite, Double Exchange, Spin-Polarised Tunneling

\section{Introduction}

Intense interest is currently shown in researching transport properties of the multicomponent compounds synthesized on the basis of elements with prominent (perovskites) and even strong (oxipnictides with iron) magnetism. This results from the fact that the properties of the above compounds are defined and are capable of being controlled by a whole complex of the mechanisms of interaction, including exchange mechanisms, which correlate behavior of charge carriers. At that, the factor of sample polycrystallinity which is characteristic, in particular, of the granulated composites of ceramic type, brings its own share in even greater variety of properties. Thus, during the study of magnetoresistance in ceramic manganites, large magnetoresistance was found in low fields below the ferromagnetic transition temperature $T_{C}$ [1-4], while magnetoresistance of single crystals is either quite small or completely absent under the same conditions. At present, there exists an understanding that the character of conductivity of granulated systems results from two sources: in-granule (intrinsic) physical mechanisms of conductivity and mechanisms of intergranule interactions; this understanding exists in spite of the fact that samples of granulated systems, even those synthesized by the same solid-state technology, are capable of being different in the character of the distribution of granules, in their size, as well as in the percolation character of current flow.

Here, we study certain properties of granulated cobaltites with $\mathrm{La}_{1-\mathrm{x}} \mathrm{Sr}_{\mathrm{x}} \mathrm{CoO}_{3}$ (hereafter LSCO) composition, a typical representative of the family of hole-doped compounds based on transition metal oxides with perovskite structure. In this family, the effects of correlation of conduction electrons operated by doping can be treated as intrinsic conductivity mechanisms. The level of doping sets the size and sign of exchange interactions as parameters of correlation between local ionic magnetic moments and spins of delocalized conduction electrons [1-3]. These quantities define the type of interaction, including ferromagnetic, when electrons are transmitted in accordance with the scheme of double exchange [4]. Other intrinsic mechanisms of conductivity may include in-granule electron transport mechanisms of the Mott type arising under the conditions of aperiodical field of force in a general sense [5]. 
Extrinsic effects are, in particular, the effects of intergranule electrostatic interactions similar to the Coulomb blockade. In such effects, a model of hopping conductivity is regularly used for the description of the process of moving electrons between the pairs of granules by way of tunneling [6,7]. The idea is based on the fact that in order to generate an electron in a neutral granule and carry it over to the next granule which was originally neutral, it is necessary to break a certain energy barrier connected with Coulomb repulsion and characterized by certain electrostatic charging energy $E_{C}$ and/or to overcome an exchange interaction by $E_{m}$ in value if the granules possess noncompensated spontaneous magnetic moment. These reasons provided the basis for various models of tunneling, such as the model of intergranule spin-dependent Coulomb gap [8] or the model of spinpolarized tunneling between magnetic granules [9].

There is no doubt that the model of intergranule tunneling reflects the physical essence of intergranule interactions, but it can widely vary in connection with concrete samples as objects in which the probability of tunneling depends, among other issues, on "structural" (distribution patterns based on the size and form of granules) and "geometrical" (the size of granules and distance between them) factors, while an average probability over an ensemble of granules on the whole which is defining conductance depends on magnetic ordering of the ensemble [7].

In general, one may expect that the character of conductivity of granulated systems will be defined by the joint contribution from the mechanisms connected both with the intrinsic (in-granule) and extrinsic (intergranule) conductivity parts which competition, in particular, could manifest itself as low-temperature resistance minimum $[10,11]$.

As this effect in bulk perovskite systems has been studied, actually, only on manganites, it appeared to be interesting (and important from the technological point of view) to continue its study in other granular magnetic composites, in particular, in bulk ceramic cobaltite, which was done for the first time by our team.

\section{Experimental Procedure}

The compound LSCO was synthesized by a standard three-phase technology [10]. Radiographic research confirmed the formation of perovskite structure with the rhombohedrically deformed unit cell (space group R3c) (Figure 1). The data from X-ray phase and microstructural analyses revealed that the synthesized ceramics were monophase, with the average size of grains (according to Sherrer) of the order of a micron.

The samples were cut out in the shape of rectangular

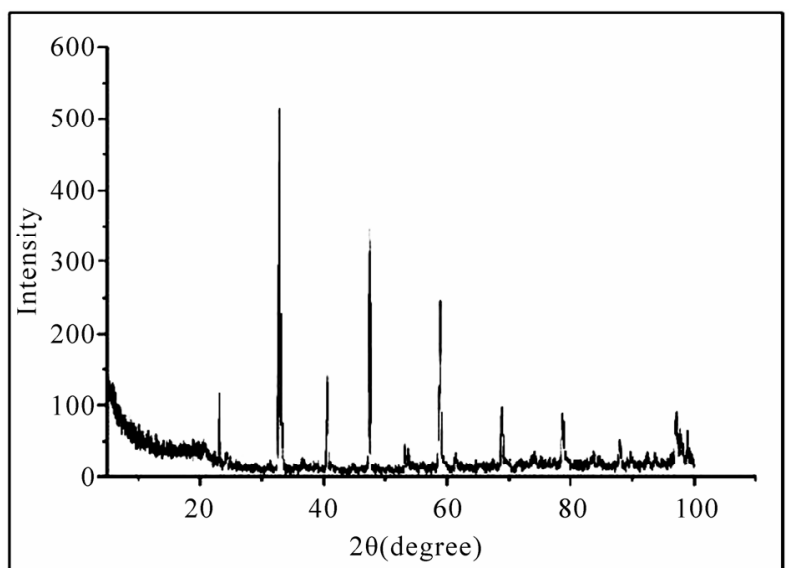

Figure 1. X-ray diffraction pattern of cobaltite $\mathbf{L a}_{0,8} \mathbf{A g}_{0,2}$ $\mathrm{CoO}_{3}$.

parallelepipeds with the sizes close to $0.15 \times 0.3 \times 0.5$ $\mathrm{cm}^{3}$. Contact pads for current and potential probes were put using the method of ultrasonic soldering of ultrapure In. Voltage measurements were carried out by 4-probe method at the constant stabilized current flow. The temperature was measured by platinum thermometer TP 018 -03 , which was additionally calibrated below $13 \mathrm{~K}$ at the temperature of superconducting transition of lead and at liquid helium temperature. Combined error of the measurements at intermediate temperatures "liquid heliumliquid nitrogen" and "liquid nitrogen-room temperature" was defined, mainly, by the quality of temperature stabilization and in all cases did not exceed $1 \%$.

\section{Results and Discussion}

In Figure 2 shown are the temperature-dependent resistances of the synthesized samples of La cobaltites differently doped by strontium, without or with silver impurity. These dependences are normalized by the resistance at room temperature. It is clearly visible that it is precisely the hole doping by strontium that drastically increases the conductivity of cobaltite LCO with perovskite structure which (the conductivity) initially is characterized by temperature behavior typical of a semiconductor. Thus, comparing the curves 1 and 2 one can see that at approximately equal concentration of silver, the resistance sharply decreases (at $100 \mathrm{~K}$ by a factor of $10^{4}$ ) only at the introduction of strontium.

Moreover, with reducing the quantity of silver but while entering at the same time a certain quantity of $\mathrm{Sr}$ with the replacement factor $x=0.35$, the composite shows metal behavior at a major portion of a temperature scale. When the temperature decreases down to 30-60 K, the minimum is observed and the behavior becomes of non-metallic type (compare curves 2 and 3 in Figure 2, 
as well as the curve 1, Figure 2, for LACO without $\mathrm{Sr}$ and the curve for LSCO without Ag in Figure 3). Apparently, we have received an additional confirmation of the insignificant role of silver for the correlation of electrons in cobaltites by means of exchange processes with the participation of oxygen ions, probably, because of weak reactivity and oxidizing power of this element or at least in the composites of the chemical compound that we studied. Notice that the transition of such a type is observed in bulk samples of granulated cobaltite LSCO, with the size of grain 1 micron and the specified degree of doping by strontium, for the first time. Such transition, for example, was not observed in bulk ceramics of the

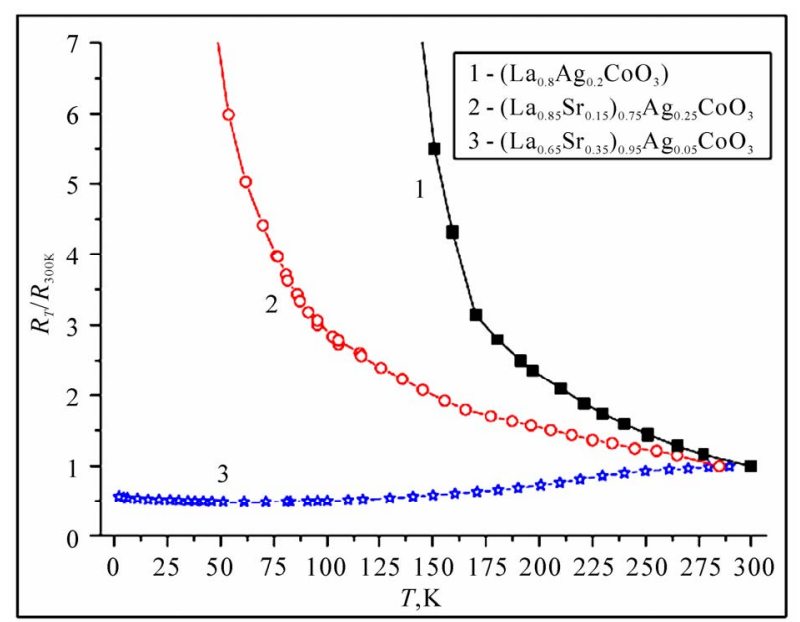

Figure 2. Resistance of hole-doped cobaltite LSACO normalized by the resistance at $300 \mathrm{~K}$ in dependence of temperature, at various doping levels. $R_{300 K}, O h m-1: 2.42$; 2 : 0.0077; 3: 0.0026 .

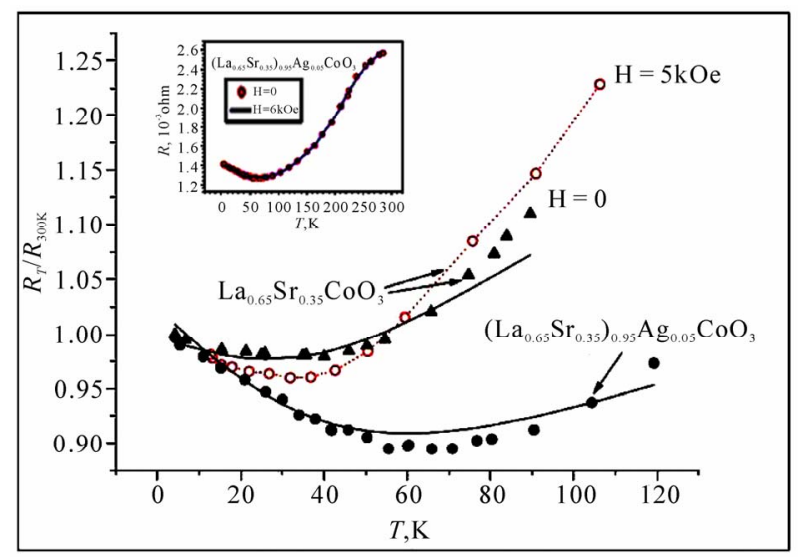

Figure 3. Minimum on the temperature dependence of the normalized resistance of hole-doped cobaltite LCO at the level of replacement of lanthanum by strontium $x \approx 0.35$. $R_{300 \mathrm{~K}}$ : for LSCO $-4.7 \times 10^{-4} \mathrm{Ohm}$; for LSACO see Caption to Figure 2. Points: experiment; solid lines: fit in accordance with (1), see text. Inset: $\mathbf{R}(\mathrm{T})_{\text {LSACO }}$ at $\mathbf{H}=\mathbf{0}$ (circles) and 5kOe (line). same cobaltite but with the size of grain $\approx 50$ micron and with $x>0.35$ [13]. It indicates that the effect is not only rather critical to the level of hole doping, which in general is characteristic of conductivity of perovskite compounds based on oxides of transition metals, but also to structural characteristics such as the size and density of distribution of grains.

Part of the resistance curves (normalized for the resistance at $300 \mathrm{~K}$ ) with a minimum corresponding to the transition from metal to non-metal behavior of conductivity is presented in Figure 3. The presence of the minimum, as well as of any extremum, on the $R(T)$ dependences means the existence in corresponding temperature regions of competing scattering mechanisms or of electron correlation with contributions into conductivity comparable in value. This allows us to apply for the explanation of this phenomenon those theoretical concepts the use of which for the description of experimental data would lead to reasonable values of theoretical parameters, thereby confirming the rationality of the theoretical models themselves. Choosing the concept of two contributions, intrinsic and intergranule, we, thereby, accept that the resistance of the system is an additive sum of ingranule resistances $(R g)$ and intergranule "resistances" (resistive contributions from intergranule interactions, $\left.R_{i s p t}\right)$. Then, it is possible to represent the total resistance of the system normalized, for example, by that resistance at $300 \mathrm{~K}$, in the following way:

$$
\begin{gathered}
R(T) /\langle R(300 \mathrm{~K})\rangle=\left[G_{g}^{-1}+G_{i s p t}^{-1}\right]_{T} /\left\langle\rho_{300 K}\right\rangle ; \\
G_{g ; \text { ispt }}=\left\langle\sigma_{g ; \text { ispt }}\right\rangle,
\end{gathered}
$$

where brackets mean the averaging of corresponding contributions to the conductivity $\sigma$ over the ensemble of granules, $G$ is the conductance averaged over the sample, $\rho_{300 \mathrm{~K}}$ the resistivity at $300 \mathrm{~K}$.

As is known, the systems of (Re) SCO type (Re is a trivalent rare-earth element) are double-phase systems [3]. One of the phases, matrix $\mathrm{ReCO}$, can be characterized by a thermally activated mechanism of conductivity of semiconductor type, $\sigma_{s m}$ (for example, of the Mott type [5]) while the conductivity of the other phase is that of SCO sublattice, $\sigma_{D E}$, due to the mechanism of double ferromagnetic exchange between cobalt ions of different valence by means of the conduction electrons [4]. As a result, the intrinsic contribution of a granule into conductivity can be written as $\sigma_{g}=\sigma_{s m}+\sigma_{D E}$, where the granule conductance will make [14]:

$$
G g \propto\left(\sigma_{s m}+\sigma_{D E}\right)=\sigma_{\infty} \exp \left(-(\Delta / T)^{m}\right)+\left(\alpha e^{2} / a h\right)\left(T_{C} / T\right)
$$

Here, $\sigma_{\infty}=\sigma(T \rightarrow \infty) ; \Delta$ the band gap; $\alpha=\left|\mathrm{Co}^{4+}\right| /$ $\left|\mathrm{Co}^{3+}\right|$ is a portion of $\mathrm{Co}^{4+}$ ions, $T_{C}$ the Curie temperature, 


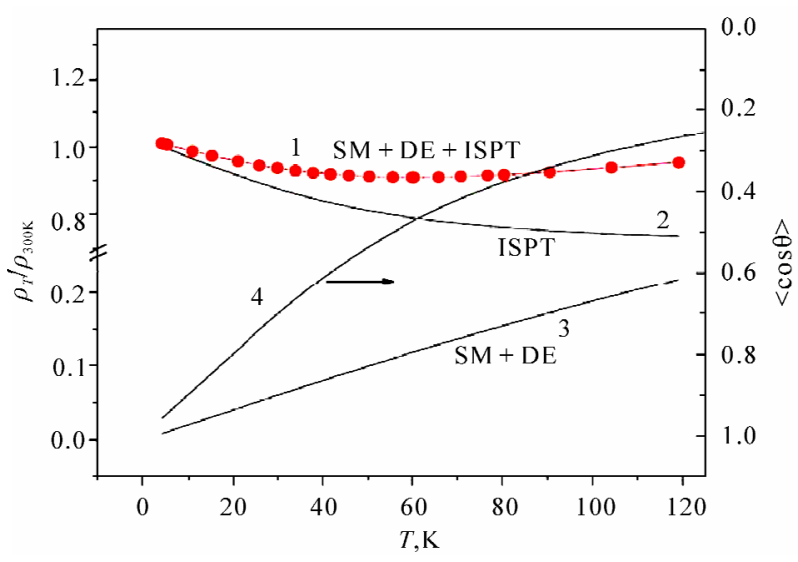

Figure 4. Normalized resistivity curve with a minimum (points) and the fit calculated from (1) (solid line 1). Shown separately are the intrinsic contribution from the granules (curve 3, SM+DE) and the intergranule contribution (curve 2, ISPT). Curve 4: spin correlation function for AFM interaction between granules.

$a$ the lattice parameter, $h$ the Planck constant, and $m$ can accept values 1 (the Arrenius law), 1/2 (when the Coulomb gap is present [7]), or $1 / 4$ (in case of variable range hopping conductance [5]). As it was discovered in [14], at low temperatures in cobaltites the mechanism of double ferromagnetic exchange prevails, causing metal behavior of conductivity at suffcient level of hole doping characterized by the $\alpha$ parameter.

An alternative contribution to the conductivity of the granulated magnetic system can be made by an extrinsic intergranule mechanism of conductivity which is based on the principle of spin-polarized tunneling [6,7]. The given model assumes that when an electron is tunnelling through a boundary between two granules with the antiparallel magnetization, it will meet a potential barrier of order of the exchange energy $J$ in size which fact reduces the probability of tunneling, i.e., the conductivity, by the factor of $e^{-J / k T}$ in comparison with the case of ferromagnetic orientation of vectors of the magnetization of granules. As it was later shown in [9], the model predicts sharp increase in the considered contribution to conductivity even in low magnetic fields $\sim 1 \mathrm{kOe}$, in case the initial (in the zero field) distribution of directions of the magnetizations of granules was random (paramagnetic). The effect should have obviously been absent if the distribution in the zero field was close to antiferromagnetic (AFM). It follows from the data in Figure 3 that the influence of the magnetic field up to $5 \mathrm{kOe}$ on conductivity of the samples with $x=0.35$ is practically absent. This fact allows us to assume AFM orientation of the magnetizations of granules in the zero field in these samples. Indications of AFM exchange between the granules are also available in $[9,15,16]$.
In [9], the expression for intergranule conductivity for two granules conditional upon the tunneling of spin polarized electrons was derived as

$$
\sigma_{\text {ispt }} \infty(1 / 2)=\left(n_{\uparrow}+n_{\downarrow}\right)^{2}\left[1+P^{2} \cos \theta\right],
$$

where polarization $P=\left(n_{\uparrow}-n_{\downarrow}\right) /\left(n_{\uparrow}+n_{\downarrow}\right)$; $n_{\uparrow, \downarrow}$ is the density of states of the electrons at Fermi - level with spin upwards and downwards, accordingly. The expression establishes the dependence of intergranule conductivity on the $\theta$ angle between the directions of magnetization of the neighboring granules. Averaging (3) over $\cos \theta$ and over probabilities of tunneling between pairs of two nearest neighbors (so-called $n-n$ model) for the whole ensemble of the system granules, as well as accounting for the temperature dependence of probability of tunneling of thermoactivated charge carriers and AFM interaction of granules $[6,7,11,16-18]$ allows us to present intergranule conductance as follows

$$
G_{\text {ispt }}=\left\langle\sigma_{\text {ispt }}\left(0<T<T_{C}\right)\right\rangle=\left[\rho_{n-n}(U)\right]^{-1}\left[1-P^{2}\langle\cos \theta\rangle\right]
$$

where $U$ is the height of intergranule potential barrier; $\rho_{n-n}(U)=\rho_{0}+\beta T^{3 / 2}[17,18] ;<\cos \theta>=[\operatorname{coth}(J / T)-$ $\left.(J / T)^{-1}\right]$ the spin correlation function $<\boldsymbol{S}_{1} \cdot \boldsymbol{S}_{2}>[11,19]$.

Combining (2) and (4), in accordance with (1), we can get the total (normalized) resistance of the sample. Assuming that the temperature behavior of intrinsic conductivity of granules in cobaltites can be of rather general character, in (2) we have kept the values of a band gap $\Delta=100 \mathrm{~K}$ in $\sigma_{s m}$ and of the temperature of AFM ordering $T_{C}=160 \mathrm{~K}$ the same, as been received at the description of low-temperature part of $R(T)$ for Er cobaltites in [14]. Besides, assuming the similarity of magnetic properties of cobaltites with close stoichiometry, we used a uniform value of exchange energy $J$ for the analyzed samples. It has actually narrowed the number of varied fitting parameters to two, namely, $\rho_{0}$ and $\beta$. This can easily be seen from the Table 1 where the main parameters obtained for the curves fitted to the experimental data are presented. The concurrence between calculated and experimental data in the region of resistance minimum for the analyzed samples gives ground for the conclusion that the intrinsic conductivity of granules in different samples of compositions with close stoichiometry is defined by practically universal mechanisms of transport (as the values of $\alpha$ and $\Delta$ can be set invariable), while the position and the depth of the minimum, all other conditions being equal, depend mainly on the probability of intergranule spin-polarized tunneling characterized by the intergranule resistance $\rho_{\mathrm{n}-\mathrm{n}}(U)$, i.e., on the values of $\rho_{0}$ and $\beta$. Though we used $\rho_{\mathrm{n}-\mathrm{n}}(U)$ in the form suggested in $[17,18]$, however, the experimental 
Table 1. The main parameters obtained for the curves fitted to the experimental data.

\begin{tabular}{ccccccc}
\hline & $\rho_{300 \mathrm{~K}}(\mathrm{Ohm} \cdot \mathrm{cm})$ & $\rho_{0}(\mathrm{Ohm} \cdot \mathrm{cm})$ & $\alpha$ & $\beta\left(\mathrm{Ohm} \cdot \mathrm{cm} \cdot \mathrm{K}^{-3 / 2}\right)$ & $P^{2}$ & $J(\mathrm{~K})$ \\
\hline $\mathrm{LaSrAgCoO}$ & $1.95 \cdot 10^{-3}$ & $1.68 \cdot 10^{-4}$ & $\approx 1$ & 0.05 & 0.37 & 100 \\
$\mathrm{LaSrCoO}$ & $3.9 \cdot 10^{-3}$ & $0.8 \cdot 10^{-4}$ & $\approx 1$ & 0.08 & 0.35 & 100 \\
\hline
\end{tabular}

curve actually reveals stronger dependence of $\rho_{\mathrm{n}-\mathrm{n}}(U)$ on temperature than when considering only a spin-wave term $\sim T^{3 / 2}$. Probably it is necessary to also consider the temperature dependence of the unbalance in the density of states for carriers with alternate spins.

\section{Conclusions}

In conclusion, the conductivity of bulk granulated cobaltites $\mathrm{La}_{1-\mathrm{x}} \mathrm{Sr}_{\mathrm{x}} \mathrm{CoO}_{3}$ was studied with the size of granules of order of 1 micron and the replacement rate of lanthanum by strontium $x \approx 0.35$. The transition «metal nonmetal» in conductivity is observed at temperatures below the temperature of magnetic transition, presumably connected with AFM ordering of the magnetization of granules. The analysis of the observed phenomenon is carried out based on allowing for in-granule mechanism of the Zener double exchange and intergranule mechanism of spin-polarized tunneling between the nearest neighbors with AFM exchange interaction. The existence of AFM interaction of granules is additionally confirmed by the fact that the value of conductivity is insensitive to low external magnetic field (up to $5 \mathrm{kOe}$ ). Microscopic parameters of double exchange and spin-polarized tunneling are estimated.

\section{References}

[1] A. Georges, G. Kotliar, W. Krauth, and M. J. Rozenberg, Reviews of Modern Physics, Vol. 68, 1996, p. 13.

[2] V. M. Loktev and Yu. G. Pogorelov, Low Temperature Physics, Vol. 26, 2000, p. 171.

[3] Yu. A. Izyumov, Yu. N. Skryabin, Physics-Uspekhi, Vol. 44, 2001, p. 109.

[4] C. Zener, Physical Review, Vol. 82, 1951, p. 403.

[5] N. F. Mott, E. A. Davis, "Electron Processes in NonCrystalline Materials", Clarendon Press, Oxford, 1979.
[6] P. Sheng, B. Abeles and Y. Arie, Physical Review Letters, Vol. 31, 1973, p. 44.

[7] J. S. Helman and B. Abeles, Physical Review Letters, Vol. 37, 1976, p. 1429.

[8] J. M. D. Coey, A. E. Berkowitz, L. Balcells, F. F. Putris, and A. Barry, Physical Review Letters, Vol. 80, 1998, p. 3815.

[9] P. Raychaudhuri, K. Sheshadri, P. Taneja, S. Bandyopadhyay, S. Chaudhary and S. B. Roy, Physical Review, Vol. 59, 1999, p. 3919.

[10] M. Garsia-Hernandez, F. Guinea, A. de Andres, J. L. Martinez, C. Prieto and L. Vazquez, Physical Review, Vol. 61, 2000, p. 9549.

[11] M. I. Auslender, E. Rozenberg, A. E. Kar'kin, B. K. Chaudhuri and G. Gorodetsky, Journal of Alloys and Compounds, Vol. 326, 2001, p. 81.

[12] A. G. Gamzatov, A. B. Batdalov, O. V. Melnikov, O. Yu. Gorbenko, Izvestiya RAN, Seriya Fizicheskaya, Vol. 73, 2009, p. 1377.

[13] B. I. Belevtsev, N. T. Cherpak, I. N. Chukanova, A. I. Gubin, V. B. Krasovitsky and A. A. Lavrinovich, Journal of Physics: Condensed Matter, Vol. 14, 2002, p. 2591.

[14] Yu. N. Chiang, V. Ph. Khirnyi, O. G. Shevchenko and A. A. Kozlovskii, Low Temperature Physics, Vol. 35, 2009, p. 876.

[15] A. E. Kar'kin, D. A. Shulyatev, A. A. Arsenov, et al., Zhurnal Eksperimentalnoii i Teoreticheskoii Fiziki, Vol. 89, 1999, p. 9358.

[16] E. Rozenberg, M. I. Auslender, I. Felner and G. Gorodetsky, Journal of Applied Physics, Vol. 88, 2000, p. 2578 .

[17] N. Zhang, F. Wang, W. Zhong and W. Ding, Solid State Communications, Vol. 107, 1998, p. 417.

[18] N. Zhang, W. Ding, W. Zhong, D. Xing and Y. Du, Physical Review, Vol. B 56, 1997, p. 8138.

[19] O. Ciftija, M. Luban, M. Auslender and J. H. Luscombe, Physical Review, Vol. B 60, 1999, p. 10122. 